\title{
Enhancement of oxygen reduction at Co-porphyrin catalyst by supporting onto hybrid multi-layered film of polypyrrole and polyoxometalate-modified gold nanoparticles
}

\author{
Sylwia Zoladek ${ }^{1}$ - Iwona A. Rutkowska ${ }^{1}$. Magdalena Blicharska ${ }^{1}$. \\ Katarzyna Skorupska ${ }^{1} \cdot$ Pawel J. Kulesza ${ }^{1}$
}

Received: 16 November 2015 / Revised: 17 January 2016 / Accepted: 19 January 2016 / Published online: 22 February 2016

(C) The Author(s) 2016. This article is published with open access at Springerlink.com

\begin{abstract}
Three-dimensional multi-layered films (on glassy carbon) composed of networks of polyoxometallate $\left(\mathrm{PMo}_{12} \mathrm{O}_{40}{ }^{3-}\right)$-modified gold nanoparticles linked together through the alternately deposited ultra-thin layers of polypyrrole have served as active supports for Co-porphyrin catalytic centers. The hybrid organic-inorganic films (supports) have been prepared by using the layer-by-layer approach. The fact that polyanionic (phosphomolybdate) adsorbates on gold nanoparticles are attracted by positively charged sites of conducting polymer (polypyrrole) structures leads to the stabilizing effect and facilitates distribution of Au nanostructures. The systems have been characterized using scanning electron microscopy, as well as with chronoamperometric and voltammetric techniques. By supporting Co-porphyrin centers onto the hybrid film of the polymer-linked phosphomolybdate-stabilized gold nanoparticles, significant electrocatalytic enhancement effects (namely voltammetric current increases) have been observed during the electroreduction of oxygen in acid medium relative to a standard response of the simple porphyrin deposit on glassy carbon measured under analogous conditions. Among important issues is the high activity of the hybrid film (support) itself toward the reductive decomposition of hydrogen peroxide to water. When it comes to performance of the Co-porphyrincontaining system, it is reasonable to expect that the $\mathrm{O}_{2}$ reduction process is initiated at Co-porphyrin catalytic sites (two-
\end{abstract}

Dedicated to Professor Jose Zagal on occasion of his 65th birthday.

Pawel J. Kulesza

pkulesza@chem.uw.edu.pl

Faculty of Chemistry, University of Warsaw, Pasteura 1, PL-02-093 Warsaw, Poland electron reduction to $\mathrm{H}_{2} \mathrm{O}_{2}$ ) and continued (two-electron reduction to $\mathrm{H}_{2} \mathrm{O}$ ) at the hybrid film containing gold nanoparticles dispersed within the highly porous cauliflower-like structures of polypyrrole multi-layers. While the gold networks facilitate charge distribution within the hybrid electrocatalytic film, non-covalent $\pi-\pi$ interactions of porphyrin rings with polypyrrole interlayers and charge transfers between negatively charged $\left(\mathrm{PMo}_{12} \mathrm{O}_{40}{ }^{3-}\right.$ modified) gold nanoparticles and positively charged nitrogen sites of polypyrrole could also cause synergism.

Keywords Conducting polymers $\cdot$ Polyoxometalates $\cdot$ Gold nanoparticles $\cdot$ Electrocatalysis $\cdot$ Oxygen reduction .

Hydrogen peroxide

\section{Introduction}

There has been growing interest in preparation of the platinum-free electrocatalysts for oxygen reduction, particularly with respect to potential applications in sensors and fuel cells. Among representative systems, nitrogen-coordinated macrocyclic complexes of transition metals, such as iron or cobalt, were demonstrated to act as promising catalysts for oxygen reduction [1-5]. It was also established that most of the monomeric cobalt porphyrins reduce oxygen via the twoelectron pathway suffering from the formation of hydrogen peroxide as the undesirable intermediate product $[6,7]$. To overcome this obstacle, chelate complex catalysts were introduced onto porous carbon supports and subjected to heattreatment (pyrolysis) $[8,9]$. Other studies showed that carbon nanoparticles modified with a cobalt ions and supported within conducting polymer film of polypyrrole, polyaniline or poly-(3-methylthiophene) films behaved as good electrocatalysts for the reduction of $\mathrm{O}_{2}$ [10]. Moreover, the 
cobalt porphine, protoporphyrin IX and 5,10,15,20tetramethylporphyrin, were successfully employed to drive efficiently the reduction of oxygen $[5,11]$. Some reports postulated high catalytic activity of face-to-face porphyrins toward the oxygen reduction reaction to water [12]. Another possible approach to achieve high activity was to utilize porphyrin catalysts distributed within matrices of carbon nanotubes (CNTs) and to explore formation of conjugative structures and the existence of $\pi-\pi$ electronic and hydrophobic interactions [13]. It was also postulated that the coexistence of cobalt porphyrin and tungsten oxide together with dispersed carbon nanotubes led to the enhancement effect promoting the efficient oxygen reduction. This phenomenon was attributed to the formation of highly conductive hydrogen tungsten oxide bronzes capable of inducing the reduction of hydrogen peroxide intermediate [13]. In addition, the presence of monolayer-type films of polytungstates at the electrocatalytic interface led to the improvement of the systems' catalytic activities. For example, chemisorption of polyoxometalates of molybdenum and tungsten on platinum catalysts resulted in the enhancement effect without blocking appreciably the active sites of Pt [14]. Another promising approach involved admixing Pt nanoparticles with the phosphododecamolybdate or phosphododecatungstate cesium salt which acted as co-catalyst, providing a proton-rich environment in the vicinity of the Pt centers $[15,16]$. Fabrication of three-dimensional (layer-by-layer) arrangements composed sequentially of ultra-thin layers of phosphotungstate-protected platinum nanoparticles and polyaniline to produce highly reactive catalysts toward oxygen reduction [17] should also be mentioned here.

In the present work, we propose and utilize hybrid organicinorganic support (for Co-porphyrin catalytic over-layer) in a form of the multi-layered film of three-dimensionally distributed of phosphomolybdate-modified gold nanoparticles linked with polypyrrole ultra-thin interlayers. The resulting electrocatalytic system (when fabricated on the inert glassy carbon electrode substrate) shows an improved performance for oxygen reduction relative to the simple Co-porphyrin deposit on glassy carbon. The fact that negatively charged polyoxometallate-modified gold nanostructures are attracted electrostatically by positively charged domains of the polypyrrole ultra-thin layers seems to have stabilizing effect and facilitate distribution of gold nanostructures. On mechanistic grounds, the initial two-electron oxygen reduction of oxygen to hydrogen peroxide is expected to be catalyzed by Coporphyrin sites; further reaction of the reductive decomposition of undesirable $\mathrm{H}_{2} \mathrm{O}_{2}$ is induced at the hybrid organicinorganic active support, namely, network multi-layered film of gold nanoparticles linked by porous polypyrrole interlayers. Improvement of the overall conductivity at the electrocatalytic interface can also be postulated due to the presence of nanostructured gold. Keggin-type phosphomolybdate adsorbates (on gold) are believed to provide fast electron transfer (mediating) capabilities as well as exhibit acid properties leading to the increased availability and mobility of interfacial protons crucial during the effective oxygen and hydrogen peroxide reductions [18-20]. Furthermore, the "capping layers" of Keggin-type phosphomolybdates are known to undergo fast stepwise multi-electron redox processes. Having in mind the ability of interfacial Keggin units to form highly reactive $\mathrm{H}_{3}\left[\mathrm{H}_{4} \mathrm{P}\left(\mathrm{Mo}^{\mathrm{V}}\right)_{4}\left(\mathrm{Mo}^{\mathrm{VI}}\right)_{8} \mathrm{O}_{40}\right]$ heteropoly blue units which combined with the specific reactivity of Au facilitating decomposition of hydrogen peroxide, we expect that phosphomolybdate-protected gold nanoparticles promote a bifunctional electrocatalytic mechanism in the vicinity of $\mathrm{Co}$ sites. The presence of conducting polymer provides the whole hybrid with environmental stability and highly porous structure. In addition, the non-covalent $\pi$-stacking interactions of Co-porphyrin with polypyrrole layers as well as charge transfers between gold particles and the polypyrrole cannot be excluded as factors effecting oxygen reduction process.

\section{Experimental}

All chemicals were commercial materials of the highest available purity, and they were used as received. Hydrogen tetrachloroaurate(III) trihydrate, $\mathrm{HAuCl}_{4} \cdot 3 \mathrm{H}_{2} \mathrm{O}(>99.9 \%$ ); sodium borohydride (powder, $98 \%$ ), $\mathrm{NaBH}_{4}$; phosphomolybdic acid hydrate, $\mathrm{H}_{3} \mathrm{PMo}_{12} \mathrm{O}_{40} \cdot \mathrm{nH}_{2} \mathrm{O}\left(\mathrm{PMo}_{12}\right)$ (ACS reagent); pyrrole; and $5 \mathrm{wt} \%$ Nafion ${ }^{\circledR}$ solution were purchased from Sigma-Aldrich, and Co(III) protoporphyrin IX chloride (Frontier) was used without any further purification. The solutions were prepared from triply distilled subsequently deionized water that had been treated with a Milli-Q water purification system.

The electrochemical measurements were carried using $\mathrm{CH}$ Instruments (Austin, TX, USA) model 660B workstation in a conventional three-electrode cell configuration. Mercury/ mercury sulfate electrode $\left(\mathrm{Hg} / \mathrm{Hg}_{2} \mathrm{SO}_{4}\right)$, with a potential of approximately $670 \mathrm{mV}$ relative to a reversible hydrogen electrode (RHE), was used as a reference electrode. The counter electrode was a carbon wire. Rotating glassy carbon disk electrode of $0.1256 \mathrm{~cm}^{2}$ surface area and glassy carbon electrode (geometric area $0.071 \mathrm{~cm}^{2}$ ) were employed as working electrodes after modification with appropriate composite film. Prior to modification, the glassy carbon electrodes were activated by polishing on a cloth wetted with successive finer grade aqueous alumina slurries (grain size, 5-0.5 $\mu \mathrm{m}$ ). All measurements were carried out at room temperature $22 \pm 2{ }^{\circ} \mathrm{C}$. Oxygen and hydrogen peroxide reduction experiments were done in $0.5 \mathrm{~mol} \mathrm{dm}^{-3}$ $\mathrm{H}_{2} \mathrm{SO}_{4}$ as electrolyte solution.

Scanning electron microscopic (SEM) examinations were performed with JEOL 5200 (Japan) microscope.

The one-step-synthesis of gold nanoparticles stabilized by ultra-thin self-assembled films of Keggin-type 
phosphomolybdates, which was based on the reaction between partially reduced phosphomolybdate heteropoly blue and the gold precursor $\left(\mathrm{HAuCl}_{4}\right)$, was described in our previous reports [21-23]. Namely, $30 \mathrm{~cm}^{3}$ of $2.56 \mathrm{mmol} \mathrm{dm}{ }^{-3}$ phosphododecamolybdic acid $\left(\mathrm{H}_{3} \mathrm{PMo}_{12} \mathrm{O}_{40}\right)$ water solution was deoxygenated by bubbling with ultra-high purified argon. Then stoichiometric quantity of $0.016 \mathrm{~mol} \mathrm{dm}^{-3}$ freshly prepared solution of sodium tetrahydridoborate $\left(\mathrm{NaBH}_{4}\right)$ was added in order to create $\mathrm{H}_{3}\left[\mathrm{H}_{4} \mathrm{P}\left(\mathrm{Mo}^{\mathrm{V}}\right)_{4}\left(\mathrm{Mo}^{\mathrm{VI}}\right)_{8} \mathrm{O}_{40}\right]$ heteropoly blue under vigorous stirring. After $5 \mathrm{~min}$, the equivalent volume of aqueous $7.5 \mathrm{mmol} \mathrm{dm} \mathrm{dm}^{-3}$ chloroauric acid $\left(\mathrm{HAuCl}_{4}\right)$ solution was added to the water phase containing partially reduced phosphomolybdate heteropolyanions. The solution was mixed for $8 \mathrm{~h}$ at room temperature to ensure the reaction completeness. Excessive amounts of phosphomolybdate anions were removed by multiple centrifuging and exchanging supernatant solutions with water; the latter step was repeated at least three times until a clear and colorless supernatant solution was obtained. The final solution of the water-dispersed phosphomolybdate-stabilized gold nanoparticles had a volume of $0.5 \mathrm{~cm}^{3}$.

Three-dimensional multilayer films composed of six layers of $\mathrm{PMo}_{12}$-stabilized gold nanostructures and five ultra-thin layers polypyrrole $\left(6 \mathrm{PMo}_{12}-\mathrm{Au} \mathrm{NPs} / 5 \mathrm{PPy}\right)$ were fabricated using the layer-by-layer method according to the procedure described earlier [17, 24, 25]. First, the glassy carbon electrode was exposed to the colloidal solution of $\mathrm{PMo}_{12}-$ protected gold (Au-PMo ${ }_{12}$ ) particles for 30 min followed by rinsing with water. The pyrrole monomer was introduced onto the layer of $\mathrm{PMo}_{12}$-modified gold nanoparticles by exposing the $\mathrm{PMo}_{12}$-modified Au electrode to a $0.07 \mathrm{~mol} \mathrm{dm}^{-3}$ solution of pyrrole in $0.5 \mathrm{~mol} \mathrm{dm} \mathrm{dm}^{-3} \mathrm{H}_{2} \mathrm{SO}_{4}$ for $3 \mathrm{~min}$. Electropolymerization of the attached monomer was achieved by voltammetric potential cycling (at $50 \mathrm{mV} \mathrm{s}^{-1}$ ) from 0.1 to $1 \mathrm{~V}$ in $0.5 \mathrm{~mol} \mathrm{dm}^{-3} \mathrm{H}_{2} \mathrm{SO}_{4}$. Eight voltammetric cycles were applied in each case to complete polymerization and to produce ultra-thin conducting polymer layer. After each treatment, the electrode was thoroughly rinsed with water. By performing additional alternate immersions in the respective solutions, three-dimensional hybrid films containing $\mathrm{PMo}_{12}$ modified Au and polypyrrole were produced.

For comparison, the conventional polypyrrole layers were electrodeposited on glassy carbon electrodes by application of two voltammetric cycles within the limits from 0.1 to $1 \mathrm{~V}$ in $0.5 \mathrm{~mol} \mathrm{dm}{ }^{-3} \mathrm{H}_{2} \mathrm{SO}_{4}$ containing $0.07 \mathrm{~mol} \mathrm{dm}^{-3}$ solution of pyrrole.

The ink of phosphomolybdate-protected gold nanostructures $\left(\mathrm{Au}-\mathrm{PMo}_{12} \mathrm{NPs}\right)$ was prepared according to the following procedure. First, $200 \mu \mathrm{l}$ of the colloidal suspension of Au-PMo ${ }_{12}$ NPs was dispersed in $600 \mu \mathrm{l}$ of ethanol $(>99.9 \%)$ and subsequently subjected to mixing using the magnetic stirrer for $6 \mathrm{~h}$. Second, $5 \mu$ of $5 \%$ Nafion (from Aldrich) was added to the received suspension, and the resulting mixture was stirred for $12 \mathrm{~h}$.

The ink containing bare phosphomolybdic acid hydrate was prepared according to analogous procedure but, instead of $\mathrm{Au}-\mathrm{PMo}_{12} \mathrm{NPs}$, bare $\mathrm{H}_{3} \mathrm{PMo}_{12} \mathrm{O}_{40}$ solid was used. Appropriate amount $(30 \mathrm{mg})$ of $\mathrm{H}_{3} \mathrm{PMo}_{12} \mathrm{O}_{40}$ heteropolyacid was subjected (together with $1600 \mu \mathrm{l}$ of ethanol) to magnetic stirring for $8 \mathrm{~h}$. Later, $100 \mu \mathrm{l}$ of $5 \%$ alcoholic Nafion solution was added to the solution containing phosphomolybdates, and stirring was continued for another $12 \mathrm{~h}$.

To perform diagnostic experiments, appropriate inks were dropped onto the surface of glassy carbon electrode and subjected to drying in air at room temperature for $60 \mathrm{~min}$. Later, the alcoholic solution of $\mathrm{Co}$ (III) protoporphyrin IX chloride (CoPPIX) was placed onto the modified glassy carbon electrode. In all cases, the CoPPIX loading was equal to $40 \mu \mathrm{g} \mathrm{cm}^{-2}$. For the sake of comparison, the catalytic films of protoporphyrin-covered polypyrrole and cobalt porphyrin within polymer-interconnected phosphomolybdate-stabilized gold were also subjected to voltammetric characterization. As a rule, the catalytic films were activated by performing 20 full voltammetric potential cycles in the range from 0.1 to $1 \mathrm{~V}$ vs. RHE (at $50 \mathrm{mV} \mathrm{s}^{-1}$ ) until steady-state currents were observed. Prior to each experiment, the electrolyte solution was bubbled for 30 min with $\mathrm{O}_{2}$ or $\mathrm{N}_{2}$, respectively. A constant nitrogen (or oxygen) flow over the solution was maintained during all measurements. Experiments were performed at room temperature $\left(22 \pm 2{ }^{\circ} \mathrm{C}\right)$.

\section{Results and discussion}

The layer-by-layer preparation of hybrid films (supports) involved formation of ultra-thin (two-dimensional like) layers of $\mathrm{PMo}_{12}$-stabilized gold nanoparticles and polypyrrole interlayers. The regular growth of voltammetric peak currents (Fig. 1a) was based on alternate immersions in the $\mathrm{PMo}_{12}$ stabilized colloidal-gold suspension followed by exposure to PPy solution (the latter step was combined with the interfacial electropolymerization of PPy as described in "Experimental" section). The procedure was consistent with the view that controlled amounts of phosphomolybdate-stabilized gold nanoparticles were introduced during alternate immersions.

In view of the literature reports [20-27], three sets of highly reversible peaks (Fig. 1a) shall be interpreted in terms of the consecutive two-electron reactions leading to the formation of partially reduced heteropoly blue phosphomolybdates according to the Eq. 1:

$\mathrm{PMo}_{12}^{\mathrm{VI}} \mathrm{O}_{40}^{3-}+n e^{-}+n \mathrm{H}^{+} \Leftrightarrow \mathrm{H}_{n} \mathrm{PMo}_{n}^{\mathrm{V}} \mathrm{Mo}_{12-n}^{\mathrm{VI}} \mathrm{O}_{40}^{3-}$

where $n$ is equal to 2,4 , or 6 . Indeed, the voltammetric characteristics of the multilayer containing gold nanoparticles and 

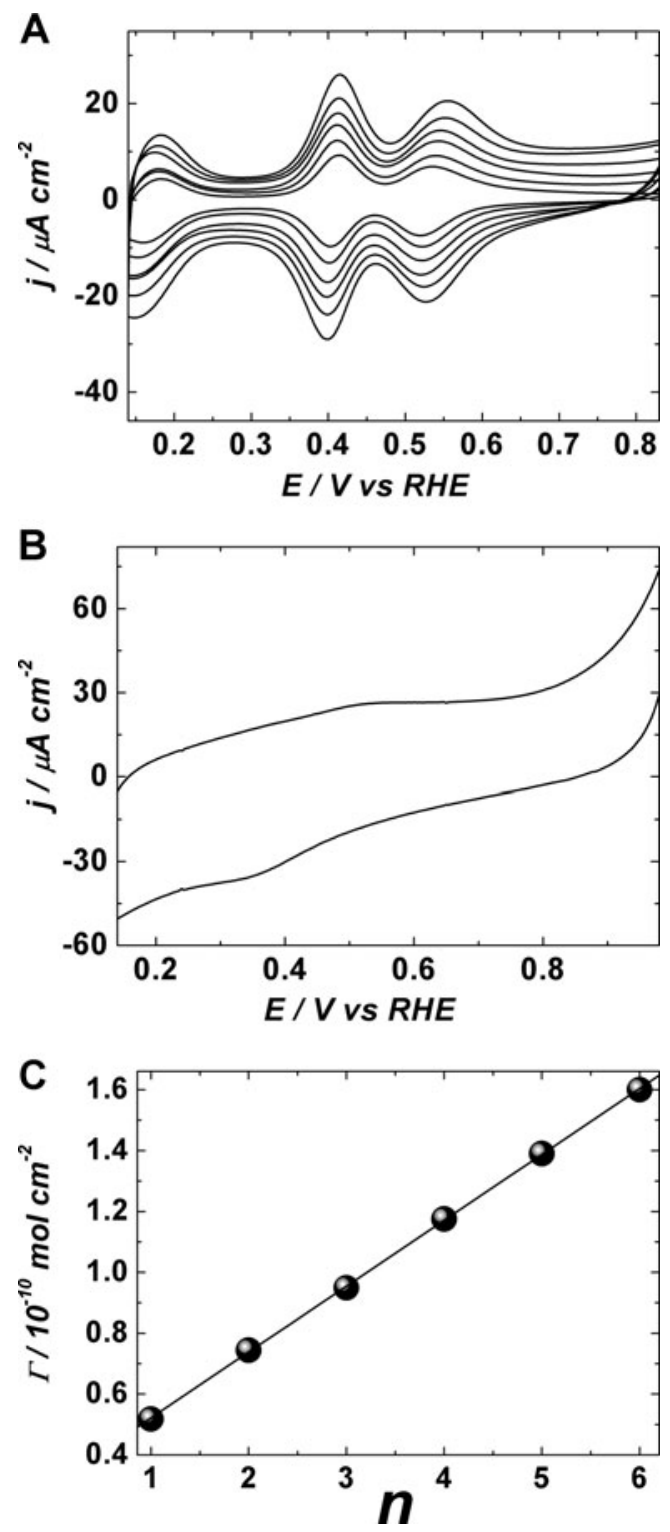

Fig. 1 a Cyclic voltammograms of the hybrid organic-inorganic multilayered films recorded after processing up to 6 cycles of alternate treatments in phosphomolybdate-modified gold nanoparticles and $0.07 \mathrm{~mol} \mathrm{dm}^{-3}$ pyrrole (in $\mathrm{H}_{2} \mathrm{SO}_{4}$ electrolyte). Each exposure in pyrrole solution was followed by external electropolymerization in the electrolyte. b Cyclic voltammetric response recorded following electrodeposition of polypyrrole. $\mathbf{c}$ Dependence of the loading of phosphomolybdates (adsorbed on gold) on a number of the alternate immersion cycles $(n)$. Electrolyte nitrogen-saturated $0.5 \mathrm{~mol} \mathrm{dm}^{-3} \mathrm{H}_{2} \mathrm{SO}_{4}$. Scan rate $50 \mathrm{mV} \mathrm{s}^{-1}$

polypyrrole become dominated by the redox reactions mixedvalent Mo (VI, V) heteropoly blue structures following incorporation of anionic $\mathrm{Au}-\mathrm{PMo}_{12} \mathrm{NPs}$. On the other hand, the conducting polymer (polypyrrole) interlayers tend to contribute to the double-layer charging (increased background currents). For comparison, a typical response of the conventional polypyrrole film is illustrated in Fig. 1b. Here, it is noteworthy that sizeable increases of voltammetric currents are observed at potentials higher than $0.85 \mathrm{~V}$, i.e., outside of the potential range where the hybrid multi-layered film is studied (Fig. 1a). The systematic formation of the multi-layered film has also been apparent from the linear dependence (Fig. 1c) of film loadings, expressed in terms of the actual surface coverages $(\Gamma)$ of polyoxometallate-stabilized Au nanoparticles (calculated from the charge under the second reduction peak at about $0.4 \mathrm{~V}$ in Fig. 1a) [28] on a number of the alternate immersion cycles.

The hybrid polypyrrole-linked gold networks are not expected to exhibit appreciable electrocatalytic activity toward oxygen reduction but possibly during electroreduction of hydrogen peroxide (the undesirable $\mathrm{O}_{2}$-reduction intermediate). The data in Fig, 2a, b refer to electrocatalytic activities (toward $\mathrm{H}_{2} \mathrm{O}_{2}$ ) of the hybrid films of various loadings of phosphomolybdate-protected gold nanoparticles and polypyrrole interlayers on glassy carbon electrode. The question arises whether immobilization of polypyrrole interlayers on phosphomolybdate-protected gold nanoparticles blocks or enhances their activities. To comment on the influence of polypyrrole interfacial electropolymerization on the electrocatalytic activity of gold, we have recorded linear sweep voltammograms in the presence of $10 \mathrm{mmol} \mathrm{dm}^{-3} \mathrm{H}_{2} \mathrm{O}_{2}$ in $0.5 \mathrm{~mol} \mathrm{dm}^{-3}$ $\mathrm{H}_{2} \mathrm{SO}_{4}$ following the application of alternate treatments in the colloidal solution of $\mathrm{PMo}_{12}$-protected gold nanoparticles and the cationic pyrrole solution (coupled with its interfacial electropolymerization). Figure $2 \mathrm{~b}$ illustrates the dependencies of the hydrogen peroxide reduction currents on the number of the alternate immersion cycles (1-6). The increases of the $\mathrm{H}_{2} \mathrm{O}_{2}$ peak reduction currents, combined with the positive shifts of onset potentials for $\mathrm{H}_{2} \mathrm{O}_{2}$ reduction, have been observed (Fig. 2a) during systematic growth of the hybrid film through alternate treatments described above. Furthermore, by plotting the current densities characteristic of the hydrogen peroxide reduction against the number of $\mathrm{PMo}_{12}-\mathrm{Au} \mathrm{NP} /$ polypyrrole bilayers, linear dependencies have been obtained (using the data obtained at distinct potentials in the range from 0.1 to $0.5 \mathrm{~V}$ ). This result can be rationalized in terms of the lack of blocking of surfaces of phosphomolybdate-protected Au NPs; furthermore, polypyrrole interlayers seem to enhance electrocatalytic phenomena. On the whole, the interfacial modifications of gold nanoparticles by $\mathrm{PMo}_{12} \mathrm{O}_{40}{ }^{3-}$ polyanions provide platforms for self-assembling, and they permit buildup of three-dimensional hybrid organicinorganic catalytic architectures.

Additional diagnostic experiments involved chronoamperometric monitoring of current increases (Fig. 3a) upon consecutive additions of $0.05 \mathrm{mmol} \mathrm{dm}^{-3}$ hydrogen peroxide and application of the working potential of $0.15 \mathrm{~V}$ (vs. RHE). The steady-state signals were produced fairly rapidly, namely, within less than $3 \mathrm{~s}$, and the established current responses were stable and reproducible within $3 \%$ (based on at least 10 independent experiments). Figure $3 \mathrm{~b}$ illustrates the dependence (calibration curve) of the steady- 

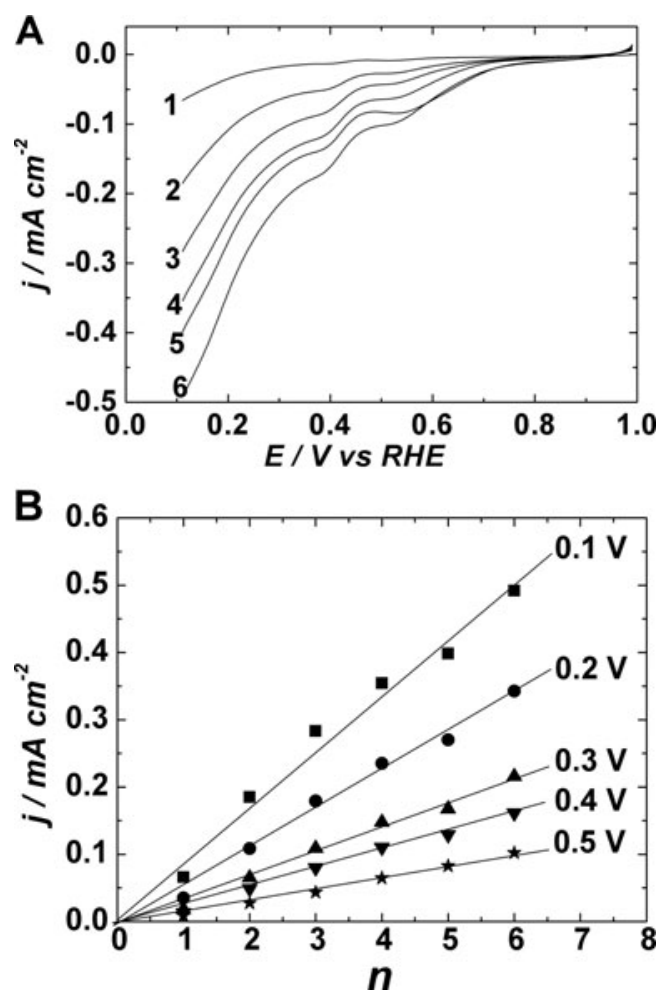

Fig. 2 a Linear sweep voltammograms for the electrocatalytic reduction

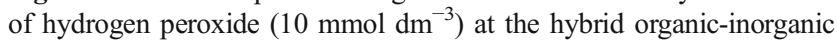
film composed of different numbers of $\mathrm{Au}-\mathrm{PMo}_{12} /$ polypyrrole bilayers following application of one (1), two (2), three (3), four (4), five (5), and six $(6)$ cycles of alternate treatments in the colloidal solution of $\mathrm{PMo}_{12^{-}}$ modified Au NPs and $0.07 \mathrm{~mol} \mathrm{dm}^{-3}$ pyrrole (in $0.5 \mathrm{~mol} \mathrm{dm}^{-3} \mathrm{H}_{2} \mathrm{SO}_{4}$ ). b Dependencies of the hydrogen peroxide reduction currents on a number $(n)$ of the alternate immersion cycles (1-6), recorded at different potentials: $0.1,0.2,0.3,0.4$, and $0.5 \mathrm{~V}$ (vs. RHE). Electrolyte de-aerated $0.5 \mathrm{~mol} \mathrm{dm}^{-3} \mathrm{H}_{2} \mathrm{SO}_{4}$. Scan rate $10 \mathrm{mV} \mathrm{s}^{-1}$

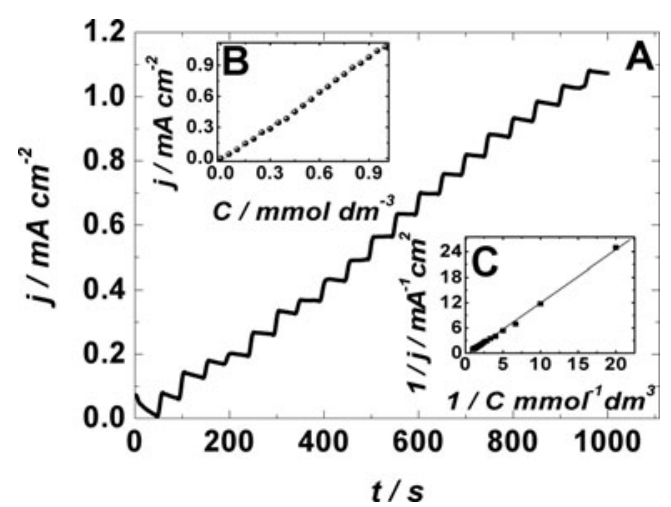

Fig. 3 Chronoamperometric results for the reduction of hydrogen peroxide obtained at the rotating disk glassy carbon electrode covered with the multi-layered hybrid film of polypyrrole-linked phosphomolybdate-modified gold nanoparticles upon application of $0.15 \mathrm{~V}$ (vs RHE). (A) Responses recorded upon sequential additions of $0.05 \mathrm{mmol} \mathrm{dm}^{-3}$ hydrogen peroxide. (B) Calibration curve plotting the catalytic current densities versus the hydrogen peroxide concentrations. (C) The double reciprocal dependence plotted using the data of the calibration curve. Electrolyte de-aerated $0.5 \mathrm{~mol} \mathrm{dm}^{-3} \mathrm{H}_{2} \mathrm{SO}_{4}$. Rotation rate $2000 \mathrm{rpm}$ state chronoamperometric current on the hydrogen peroxide concentration. The linear range of response was operative

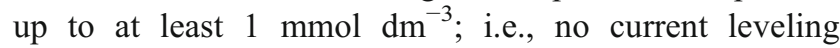
(saturation) was found in the investigated range of $\mathrm{H}_{2} \mathrm{O}_{2}$ concentrations. The response was characterized by sensitivity equal to $1.14 \mathrm{~mA} \mathrm{~cm} \mathrm{mmol}^{-1} \mathrm{dm}^{3}$ and the high correlation coefficients, $>0.996$. Figure $3 \mathrm{c}$ illustrates a linear plot of the reciprocals of current densities on the reciprocals of concentrations with effectively zero intercept. By referring to the well-known Michaelis-Menten kinetics analysis (proposed together with the Lineweaver-Burk equation for enzymatic kinetics in bioelectrochemistry [29, 30]), extrapolation to the infinitely high concentration would not permit determination of the maximized electrocatalytic current density (kinetic parameter) because, in the range of concentrations studied, it would become infinitely high. In other words, the electrocatalytic system is well-behaved, and the linear concentration dependence implies the uncomplicated pseudo-first-order-type kinetic mechanism. Thus, it can be concluded that the hybrid organic-inorganic multi-layered film composed of polyoxometallate-modified gold nanoparticles and polypyrrole interlayers exhibit high catalytic activity toward electroreduction of hydrogen peroxide in acid medium.

Figure 4 illustrates scanning electron microscopy (SEM) images of phosphomolybdate-modified Au nanoparticles (a), as well as submicrostructured polypyrrole films with (b), and without (c) the gold nanoparticles. It is noteworthy that our gold nanoparticles have comparable sizes, namely, diameters on the level 30-40 nm (Fig. 4a). Their presence in the hybrid film is evident from the data of Fig. 4b. Furthermore, the granular morphology of the polypyrrole deposits is clearly evident from the micrographs of Fig. 4b, c. While polypyrrole has been fabricated using the layer-by-layer approach in the case of Fig. 4b, the polymer has been conventionally electrodeposited for Fig. 4c. The polypyrrole granules of cauliflowerlike shapes having sizes ranging typically from 200 to $400 \mathrm{~nm}$ can be easily distinguished in both cases. On the whole, the polypyrrole matrix seems to be largely porous. What is even more important, polypyrrole does exist as a component of the multi-layered film (compare micrographs of Fig. 4). Upon incorporation of phosphomolybdate-modified $\mathrm{Au}$ nanoparticles into the multi-layered hybrid film (Fig. 4b), most of $\mathrm{Au}$ nanoparticles seem to be well dispersed. Most likely, the negatively charged phosphomolybdate polyanions (adsorbates on $\mathrm{Au}$ ) are attracted by positively charged structures of the conducting polymer (polypyrrole).

To comment on the influence of polypyrrole on the electrochemical identity of Co-porphyrin, the following series of voltammetric experiments have been performed. Figure 5a illustrates the cyclic voltammetric responses of (a) Coporphyrin deposited on bare glassy carbon electrode (dashed line), (b) conventional (electrodeposited) polypyrrole film on glassy carbon (dotted line), and (c) Co-porphyrin introduced 

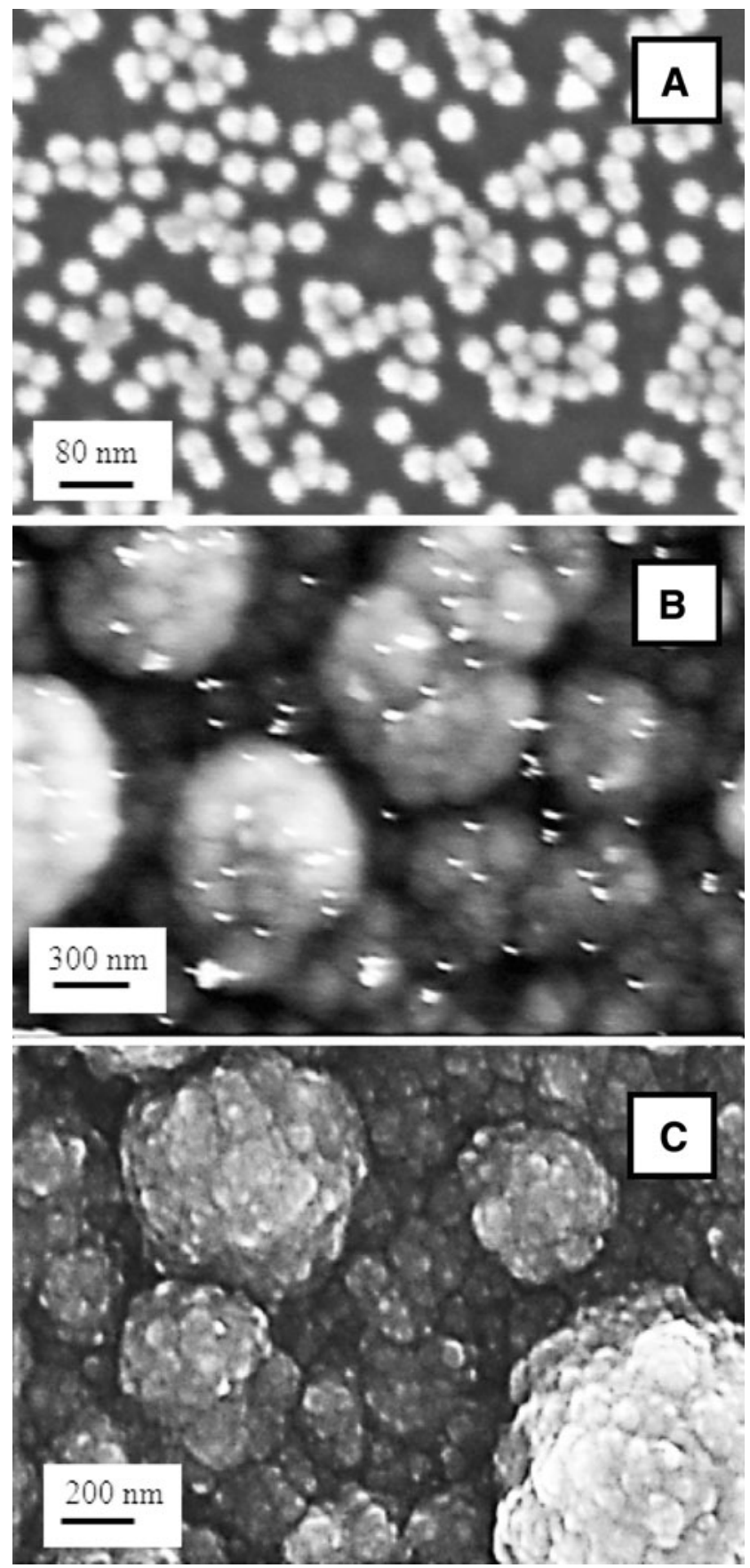

Fig. 4 SEM images of a phosphomolybdate-modified gold nanoparticles (deposited on glassy carbon); $\mathbf{b}$ hybrid multi-layered film of polypyrrolelinked phosphomolybdate-modified Au nanoparticles; c electrodeposited layer of polypyrrole

on top of the conventional polypyrrole film (on glassy carbon). While the presence of Co-porphyrin is fairly obvious from the data of curve a, the introduction of the polypyrrole underlayer (characterized by fairly large double-layer charging currents) tends to suppress the peaks originating from $\mathrm{Co}-$ porphyrin. It is not clear to what extent the well-known stabilizing interactions between the $\pi$-electron-conjugated system of cobalt porphyrin and polypyrrole [31] affect the
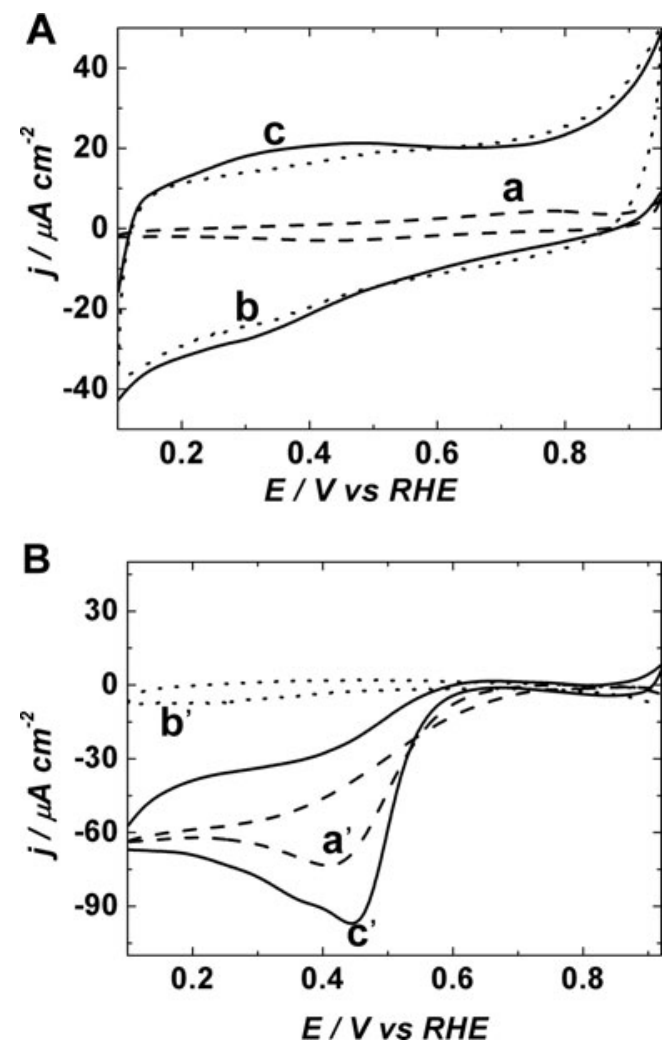

Fig. 5 a Cyclic voltammetric responses of $a$ cobalt porphyrin deposited on the bare glassy carbon substrate (dashed line), $b$ conventional electrodeposited film of polypyrrole (dotted line), and $c$ cobalt porphyrin introduced onto the electrodeposited layer of polypyrrole (solid line). b Background-subtracted oxygen reduction voltammetric responses recorded at the following systems: $a$ ' bare cobalt porphyrin (dashed line), $b^{\prime}$ electrodeposited polypyrrole film (dotted line), and $c^{\prime}$ cobalt porphyrin existing on the electrodeposited polypyrrole (solid line). Electrolyte nitrogen-saturated (for a) and oxygen-saturated (for b) $0.5 \mathrm{~mol} \mathrm{dm}^{-3} \mathrm{H}_{2} \mathrm{SO}_{4}$. Scan rate $10 \mathrm{mV} \mathrm{s}^{-1}$

voltammetric characteristics of curve $b$. When it comes to the reduction of oxygen at Co-porphyrin catalysts supported onto porous polypyrrole film, higher electrocatalytic currents and the positive shift of the peak position from 0.42 to $0.45 \mathrm{~V}$ have been observed (compare curves a' and c' in Fig. 5b).

The explanation of these phenomena should take into account the possibility of better distribution of Co-porphyrin sites upon deposition onto the polypyrrole film, improved charge distribution at the conducting-polymer-containing interface, or activating interactions between the $\pi$ electronconjugated system of Co-porphyrin and polypyrrole [32]. It is noteworthy that polypyrrole alone does not exhibit appreciable catalytic activity toward the oxygen electroreduction (curve b' in Fig. 5b).

To address the influence of the phosphomolybdatemodified gold nanoparticles on the catalytic activity of Coporphyrin, additional diagnostic voltammetric experiments have been performed. Three sets of peaks referring to the redox transitions of phosphomolybdate anions are clearly visible in the voltammetric responses of catalytic systems 
utilizing Co-porphyrin immobilized onto (a) $\mathrm{PMo}_{12}-\mathrm{Au} \mathrm{NPs}$ (Fig. 6, dotted line) and (b) the hybrid multi-layered film composed of three-dimensionally-distributed $\mathrm{PMo}_{12}-\mathrm{Au} \mathrm{NPs}$ within polypyrrole (Fig. 6, solid line). For comparison, the voltammetric pattern (c) of Co-porphyrin introduced to the "ink" of $\mathrm{H}_{3} \mathrm{PMo}_{12} \mathrm{O}_{40}$ suspended in Nafion (Fig. 6, dashed line) is provided. Here, phosphomolybdate voltammetric peaks tend to appear at potentials less negative in comparison to the analogous responses of phosphomolybdates illustrated in Fig. 6 (curves a and b). This behavior may reflect the existence of electrostatic repulsive interactions between the negatively charged heteropolyanion and Nafion structural units. Furthermore, the voltammetric response of phosphomolybdates $\left(\mathrm{PMo}_{12}\right)$ on gold (Curve a) may also be affected by the strong adsorption phenomenon. In addition, the electrostatic attractive interactions between anionic $\mathrm{PMo}_{12}$ units (adsorbed on Au NPs) and positively charged polypyrrole units should also be taken into account (Curve b).

The electrocatalytic performance of the systems considered above has been examined in the oxygen-saturated solutions. First, it should be noted that upon comparison of the voltammetric responses of the systems utilizing Coporphyrin catalyst deposited onto (a) phosphomolybdatemodified gold nanoparticles (Fig. 7, dotted line) and (c) the phosphomolybdate-based layer with Nafion (Fig. 7, dashed line), immobilization of phosphomolybdate on gold results in the enhancement effect understood in terms of the higher oxygen reduction currents and the peak potential shift toward more positive values. The appearance of the molybdate $\left(\mathrm{PMo}_{12}\right)$ redox transitions at more positive potentials relative to those characteristic of the $\mathrm{Co}$ (II)/(III) redox couple may explain higher activity toward oxygen reduction of the systems containing $\mathrm{PMo}_{12} \mathrm{O}_{40}{ }^{3-}$ together with cobalt porphyrin in comparison to pure CoPPIX [33]. The similar electrocatalytic enhancement effect has been postulated for the pyrolyzed FeNx catalyst co-existing with metallic centers characterized by redox processes existing at the relatively more positive potentials [34]. Furthermore, it is reasonable to expect that gold nanostructures acting as the powerful hydrogen peroxide reduction catalyst induce the second step in the oxygen reduction mechanism: reductive decomposition of hydrogen peroxide. The mediating (fast electron transfer) capabilities of phosphomolybdate adsorbates (also with respect to the $\mathrm{Co}^{\mathrm{III}}$ / $\mathrm{Co}^{\mathrm{II}}$ porphyrin redox transitions) should contribute to the overall enhancement effect. Because of certain analogies between Keggin-type anions and tungsten oxide, it is also likely that the partially reduced polymolybdates (e.g. $\left.\mathrm{H}_{3}\left[\mathrm{H}_{4} \mathrm{P}\left(\mathrm{Mo}^{\mathrm{V}}\right)_{4}\left(\mathrm{Mo}^{\mathrm{VI}}\right)_{8} \mathrm{O}_{40}\right]\right)$ provide monoatomic hydrogen adatoms (active sites) in similar way to hydrogen tungsten oxide bronzes $\left(\mathrm{H}_{x} \mathrm{WO}_{3}\right)$ being able to facilitate decomposition of the undesirable hydrogen peroxide intermediate $[14,17,19$, $31,35]$. The above properties should promote the bifunctional electrocatalytic mechanism for the oxygen reduction in which

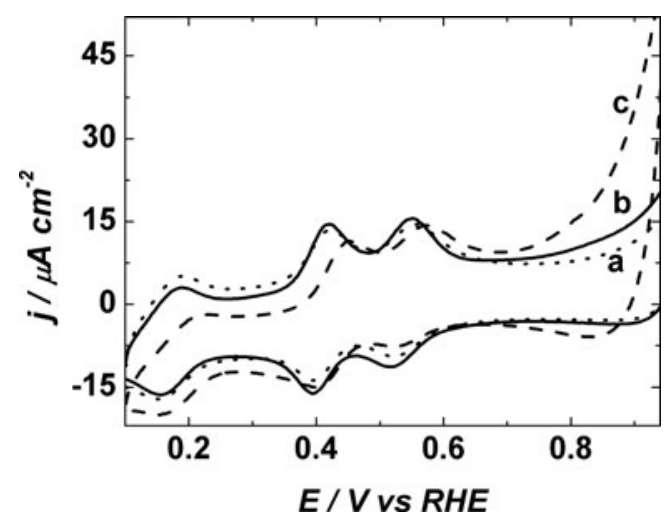

Fig. 6 Cyclic voltammetric responses of cobalt porphyrin deposited on the following supports: $a$ layer of phosphomolybdate-modified gold nanoparticles (dotted line), $b$ hybrid multi-layered film composed of $\mathrm{PMo}_{12}$-modified gold nanoparticles and ultra-thin layers of PPy (solid line), and $c$ layer of $\mathrm{H}_{3} \mathrm{PMo}_{12} \mathrm{O}_{40}$ admixed with $\mathrm{Nafion}$ (dashed line). Electrolyte nitrogen-saturated $0.5 \mathrm{~mol} \mathrm{dm}^{-3} \mathrm{H}_{2} \mathrm{SO}_{4}$. Scan rate $50 \mathrm{mV} \mathrm{s}^{-1}$

Co-porphyrin sites induce the two-electron pathway yielding predominantly $\mathrm{H}_{2} \mathrm{O}_{2}$, while $\mathrm{H}_{3}\left[\mathrm{H}_{4} \mathrm{P}\left(\mathrm{Mo}^{\mathrm{V}}\right)_{4}\left(\mathrm{Mo}^{\mathrm{VI}}\right)_{8} \mathrm{O}_{40}\right]$ together with gold nanoparticles facilitates reductive decomposition of the $\mathrm{H}_{2} \mathrm{O}_{2}$ intermediate to $\mathrm{H}_{2} \mathrm{O}$. In view of the previous important observations [36], immobilization of cobalt porphyrin sites within close vicinity of gold nanoparticles may facilitate transfer of free electrons from gold to central cobalt ions thus affecting electronic properties of the organic-metal interface and enhancing the oxygen reduction. Incorporation

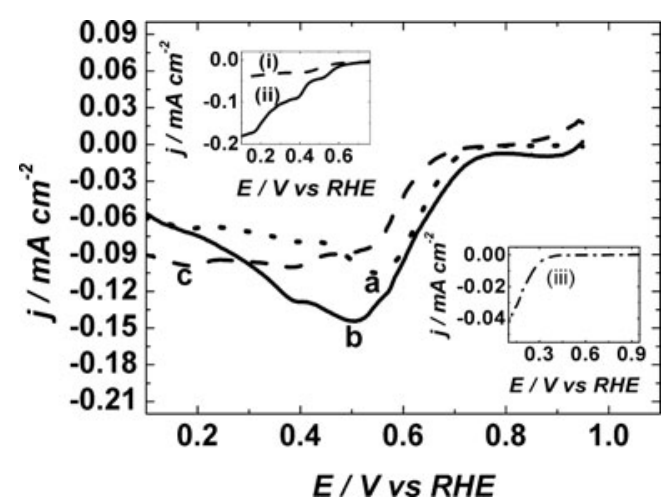

Fig. 7 Background-subtracted voltammetric responses for the reduction of oxygen recorded at the following layers: $a$ Co-porphyrin supported onto phosphomolybdate-modified Au nanoparticles (dotted line), $b$ Coporphyrin deposited at the multi-layered hybrid film composed of polypyrrole-linked three-dimensionally distributed phosphomolybdatemodified $\mathrm{Au}$ nanoparticles, and $c$ Co-porphyrin introduced onto the deposit $\mathrm{H}_{3} \mathrm{PMo}_{12} \mathrm{O}_{40}$ with Nafion (dashed line). Electrolyte $\mathrm{O}_{2}$-saturated $0.5 \mathrm{~mol} \mathrm{dm}^{-3} \mathrm{H}_{2} \mathrm{SO}_{4}$. Scan rate $10 \mathrm{mV} \mathrm{s}{ }^{-1}$. Upper inset illustrates background-subtracted voltammetric responses recorded in 2 mmol dm ${ }^{-3}$ solutions of $\mathrm{H}_{2} \mathrm{O}_{2}$ at glassy carbon electrode modified with (i) bare cobalt porphyrin (dashed line) and (ii) cobalt porphyrin immobilized onto the multi-layered hybrid film (solid line). Electrolyte de-aerated $0.5 \mathrm{~mol} \mathrm{dm}^{-3} \mathrm{H}_{2} \mathrm{SO}_{4}$. Scan rate $10 \mathrm{mV} \mathrm{s}^{-1}$. Lower inset shows the background-subtracted voltammetric response (iii) for the reduction of oxygen recorded at the multi-layered hybrid film composed of polypyrrole-linked three-dimensionally distributed phosphomolybdatemodified Au nanoparticles (dashed-dotted line) 
of the porous conducting polymer structures to the support (as components of the hybrid organic-inorganic multi-layered film containing phosphomolybdate-modified Au nanoparticles) should lead to the increase of the electrochemically active surface area. Indeed, this step has resulted in the highest catalytic activity toward electroreduction of oxygen (Fig. 7, solid line) in terms of both shifting the potential to more positive values and increasing the reduction currents (compare curve b with curves a and c). The network of phosphomolybdate-modified gold nanoparticles and polypyrrole alone (i.e. without Co-porphyrin sites) would exhibit rather poor catalytic activity toward oxygen electroreduction (curve (iii)-dashed-dotted line in inset of Fig. 7). The positive role of polypyrrole interlayers (in the hybrid support for Coporphyrin) concerns their high degree of porosity (facilitating dispersion of phosphomolybdate-modified Au nanoparticles) as well as possible interactions with the catalyst (Coporphyrin) itself. In view of the strong electron-withdrawing ability of nitrogen atoms and the electron-rich identity of gold, it cannot be excluded that the $\mathrm{N}$ atoms of polypyrrole attract electrons from gold. Indeed, the nitrogen-gold interactions and a negative shift in the $\mathrm{N} 1 s$ binding energy has been postulated on basis of the XPS measurements [37].

When returning to the feasibility of the bifunctional oxygen reduction mechanism, it should be emphasized that the system utilizing the hybrid organic-inorganic support (as for Curve b in Fig. 7) exhibits very high catalytic activity toward the hydrogen peroxide electroreduction (refer to Figs. 2 and 3). The simple comparison of the respective responses obtained at "bare" Co-porphyrin deposit and at Co-porphyrin over-coating the hybrid support (compare curves $(i)$ and (ii) in inset of Fig. 7) works strongly in favor the system utilizing the polypyrrole-linked gold nanostructures.

Finally, it is noteworthy that three-dimensional multi-layered hybrid films were stable during electrocatalytic experiments performed several (at least 10) times within 5-10 days (in the meantime, the electrodes were stored in the electrolyte under open-circuit conditions). After that period of time, the catalytic films were physically removed. The stability and durability of the modified electrodes significantly improved upon fabrication of multi-layered polypyrrole containing films with networks of phosphomolybdate-modified $\mathrm{Au}$ nanoparticles.

\section{Conclusions}

By using a model catalyst, Co-porphyrin, which is capable of inducing the less-efficient two-electron (rather than more efficient four-electron) electroreduction of oxygen (in acid medium, $0.5 \mathrm{~mol} \mathrm{dm}^{-3} \mathrm{H}_{2} \mathrm{SO}_{4}$ ), we have been able to address the importance of a support with respect to the possible enhancement effect. The combination of the polypyrrole structures within the network of three-dimensionally distributed gold (polyoxometallate-modified) nanoparticles has produced hybrid multi-layered film (support) exhibiting high activity toward electroreduction of hydrogen peroxide. Among the important features are the high reactivity of nanostructured gold, high degree of its dispersion on porous polypyrrole interlayers, good mediating (electron transfer) capabilities of phosphomolybdate adsorbates, and attractive interactions between polyanionic adsorbates and positively charged units of the conducting polymer. The observed synergism may also be influenced by non-covalent $\pi-\pi$ interactions between porphyrins rings and polypyrrole units thus affecting electron densities and in the vicinity of cobalt ions and the dynamics of their redox transitions center, leading to the improved oxygen electroreduction process. On the whole, our results imply that all the components (nanostructured gold, polypyrrole and polyoxometalates), alone or in combination, exhibit a positive effect on the activity of Co-porphyrin catalyst. Similar effects have been observed for iron phthalocyanine electrocatalysts with axial (pyridyl group) ligands anchored to multi-walled carbon nanotube carriers [38].

The enhancement effect in the oxygen reduction reaction upon utilization of the hybrid multi-layered organic-inorganic film as support for Co-porphyrin catalytic sites is fairly sizeable. This observation results most likely from the sufficiently fast kinetics of $\mathrm{H}_{2} \mathrm{O}_{2}$ reduction at the hybrid film containing both $\mathrm{Au}$ nanoparticles and polypyrrole (as shown in Figs. 3 and 7). By referring to the classical concepts of multi-step electrode reactions $[39,40]$, the second step (decomposition or reduction of $\mathrm{H}_{2} \mathrm{O}_{2}$ ) should be characterized by relatively high rate constant to observe sizeable current increases or positive potential shifts (oxygen reduction). Apparently, this is a case here but further research is needed along this line.

Acknowledgments This work was supported by the National Science Center (Poland) under Maestro Project 2012/04/A/ST4/00287. Sylwia Zoladek appreciates also support from Foundation for Polish Science (FNP) under Start Project.

Open Access This article is distributed under the terms of the Creative Commons Attribution 4.0 International License (http:// creativecommons.org/licenses/by/4.0/), which permits unrestricted use, distribution, and reproduction in any medium, provided you give appropriate credit to the original author(s) and the source, provide a link to the Creative Commons license, and indicate if changes were made.

\section{References}

1. Jahan M, Bao QL, Loh KP (2012) Electrocatalytically active graphene-porphyrin MOF composite for oxygen reduction reaction. J Am Chem Soc 134:6707-6713

2. Carver CT, Matson BD, Mayer JM (2012) Electrocatalytic oxygen reduction by iron tetra-arylporphyrins bearing pendant proton relays. J Am Chem Soc 134:5444-5447 
3. Jasinski R (1965) Cobalt phthalocyanine as a fuel cell cathode. J. Electrochem Soc 112:526-528

4. Gojkovic SL, Gupta S, Savinell RF (1999) Heat-treated iron(III) tetramethoxyphenyl porphyrin chloride supported on high-area carbon as an electrocatalyst for oxygen reduction: Part II. Kinetics of oxygen reduction. Electroanal Chem 462:63-72

5. Shi CN, Anson FC (1998) (5,10,15,20-Tetramethylporphyrinato)cobalt(II): a remarkably active catalyst for the electroreduction of O to H O. Inorg Chem 37:1037-1043

6. Kobayashi N, Janda D, Lever AP (1992) Cathodic reduction of oxygen and hydrogen peroxide at cobalt and iron crowned phthalocyanines adsorbed on highly oriented pyrolytic graphite electrodes. Inorg Chem 31:5172-5177

7. See Yu P, Yan J, Zhao H, Su L, Zhang J, Mao L (2008) Rational functionalization of carbon nanotube/ionic liquid bucky gel with dual tailor-made electrocatalysts for four-electron reduction of oxygen. J Phys Chem C 112:2177-2182

8. Okada T, Gokita M, Yuasa M, Sekine I (1998) Oxygen reduction characteristics of heat-treated catalysts based on cobalt-porphyrin ion complexes. J Electrochem Soc 145:815-822

9. Widelov A (1993) Pyrolysis of iron and cobalt porphyrins sublimated onto the surface of carbon black as a method to prepare catalysts for O reduction. Electrochim Acta 38:2493-2502

10. Martínez Millán W, Toledano Thompson T, Arriaga LG, Smit MA (2009) Characterization of composite materials of electroconductive polymer and cobalt as electrocatalysts for the oxygen reduction reaction. Int J Hydrog Energy 34:694-702

11. Song E, Shi C, Anson FC (1998) Comparison of the behavior of several cobalt porphyrins as electrocatalysts for the reduction of $\mathrm{O}$ at graphite electrodes. Langmuir 14:4315-4321

12. Chang CJ, Loh ZH, Shi C, Anson FC, Nocera DG (2004) Targeted proton delivery in the catalyzed reduction of oxygen to water by bimetallic pacman porphyrins. J Am Chem Soc 126:10013-10020

13. Dembinska B, Kulesza PJ (2009) Multi-walled carbon nanotubesupported tungsten oxide-containing multifunctional hybrid electrocatalytic system for oxygen reduction in acid medium. Electrochim Acta 54:4682-4687

14. Chojak M, Kolary-Zurowska A, Wlodarczyk R, Miecznikowski K, Karnicka K, Palys B, Marassi R, Kulesza PJ (2007) Modification of Pt nanoparticles with polyoxometallate monolayers: competition between activation and blocking of reactive sites for the electrocatalytic oxygen reduction. Electrochim Acta 52:5574-5581

15. Kolary-Zurowska A, Zurowski A, Dsoke S, Dembinska B, Zoladek S, Kiliszek M, Marassi R, Kulesza PJ (2014) Electrocatalytic properties of platinum nanocenters electrogenerated at ultra-trace levels within zeolitic phosphododecatungstate cesium salt matrices. J Solid State Electrochem 18:2993-3001

16. Dsoke S, Kolary-Zurowska A, Zurowski A, Mignin P, Kulesza PJ, Marassi R (2011) Rotating disk electrode study of Cs H PW O as mesoporous support for Pt nanoparticles for PEM fuel cells. J Power Sources 196:10591-10600

17. Kulesza PJ, Karnicka K, Miecznikowski K, Chojak M, Kolary A, Barczuk PJ, Tsirlina G, Czerwinski W (2005) Network electrocatalytic films of conducting polymer-linked polyoxometallate-stabilized platinum nanoparticles. Electrochim Acta 50:5155-5162

18. Wlodarczyk R, Chojak M, Miecznikowski K, Kolary A, Kulesza PJ, Marassi R (2006) Electroreduction of oxygen at polyoxometallate-modified glassy carbon-supported Pt nanoparticles. J Power Sources 159:802-809

19. Wlodarczyk R, Kolary-Zurowska A, Marassi R, Chojak M, Kulesza PJ (2007) Modification of Pt nanoparticles with polyoxometallate monolayers: competition between activation and blocking of reactive sites for the electrocatalytic oxygen reduction. Electrochim Acta 52:5574-5581

20. Ingersoll D, Kulesza PJ, Faulkner LR (1994) Polyoxometallatebased layered composite films on electrodes preparation through alternate immersions in modification solutions. J Electrochem Soc 141:140-147

21. Zoladek S, Rutkowska IA, Skorupska K, Palys B, Kulesza PJ (2011) Fabrication of polyoxometallatemodified gold nanoparticles and their utilization as supports for dispersed platinum in electrocatalysis. Electrochim Acta 56:10744-10750

22. Zoladek S, Rutkowska IA, Kulesza PJ (2011) Enhancement of activity of platinum towards oxidation of ethanol by supporting on titanium dioxide containing phosphomolybdate-modified gold nanoparticles. Appl Surf Sci 257:8205-8210

23. Lublow M, Skorupska K, Zoladek S, Kulesza PJ, Vo-Dinh T, Lewerenz HJ (2010) On the behaviour of Au plasmonic nanoparticles during hydrogen evolution at p-Si. Electrochem Commun 12: 1298-1301

24. Kulesza PJ, Chojak M, Karnicka K, Miecznikowski K, Palys B, Lewera A, Wieckowski A (2004) Network films composed of conducting polymer-linked and polyoxometallate-stabilized platinum nanoparticles. Chem Mater 16:4128-4134

25. Ernst AZ, Zoladek S, Wiaderek K, Cox JA, Kolary-Zurowska A, Miecznikowski K, Kulesza PJ (2008) Network films of conducting polymer-linked polyoxometalate-modified gold nanoparticles: preparation and electrochemical characterization. Electrochim Acta 53:3924-3931

26. Kuhn A, Anson FC (1996) Adsorption of monolayers of P Mo O and deposition of multiple layers of Os(bpy) ?P Mo O on electrode surfaces. Langmuir 12:5481-5488

27. Cheng L, Niu L, Gong J, Dong S (1999) Electrochemical growth and characterization of polyoxometalate-containing monolayers and multilayers on alkanethiol monolayers self-assembled on gold electrodes. Chem Mater 11:1465-1475

28. Rutkowska IA, Zoladek S, Kulesza PJ (2015) Polyoxometallateassisted integration of nanostructures of $\mathrm{Au}$ and $\mathrm{ZrO}$ to form supports for electrocatalytic PtRu nanoparticles: enhancement of their activity toward oxidation of ethanol. Electrochim Acta 162:215223

29. Berg JM, Tymoczko JL, Stryer L (2002) Biochemistry, 5 edn. W.H. Freeman and Company, New York

30. Kowalewska B, Kulesza PJ (2012) Toward more efficient bioelectrocatalytic oxidation of ethanol for amperometric sensing and biofuel cell technology. Anal Chem 84:9564-9571

31. Borzenko M, Chojak M, Kulesza PJ, Tsirlina GA, Petrii OA (2003) Platinization assisted by Keggin-type heteropolytungstates. Electrochim Acta 48:3797-3804

32. Zhou Q, Li CM, Li J, Cui X, Gervasio D (2007) Template-synthesized cobalt porphyrin/polypyrrole nanocomposite and its electrocatalysis for oxygen reduction in neutral medium. J Phys Chem C 111:11216-11222

33. Zagal JH, Recio FJ, Gutierrez CA, Zuñiga C, Páez MA, Caro CA (2014) Towards a unified way of comparing the electrocatalytic activity MN4 macrocyclic metal catalysts for $\mathrm{O}$ reduction on the basis of the reversible potential of the reaction. Electrochem Commun 41:24-26

34. Jia Q, Ramaswamy N, Hafiz H, Tylus U, Strickland $\mathrm{K}, \mathrm{Wu} \mathrm{G}$, Barbiellini B, Bansil A, Holby EF, Zelenay P, Mukerjee S (2015) Experimental observation of redox-induced Fe-N switching behavior as a determinant role for oxygen reduction activity. ACS Nano 9:12496-12505

35. Mishina ED, Tsirlina GA, Timofeeva EV, Sherstyuk NE, Borzenko MI, Tanimura N, Nakabayashi S, Petrii OA (2004) Adlayers of Keggin type polytungstate anions on platinum: negligible electrochemical signatures and manifestations of "Molecular UPD". J Phys Chem B 108:17096-17105

36. Petraki F, Peisert H, Uihlein J, Aygül U, Chassé T (2014) CoPc and $\mathrm{CoPcF} 16$ on gold: site-specific charge-transfer processes. Beilstein J Nanotechnol 5:524-531 
37. Zhang J, Liu X, Zhang L, Cao B, Wu S (2013) Reactive template synthesis of polypyrrole nanotubes for fabricating metal/conducting polymer nanocomposites. Macromol Rapid Commun 34:528-532

38. Peng Y, Li Z, Xia D, Zheng L, Liao Y, Li K, Zuo X (2015) Probing the influence of the center atom coordination structure in iron phthalocyanine multi-walled carbon nanotube-based oxygen reduction reaction catalysts by X-ray absorption fine structure spectroscopy. J Power Sources 291:20-28

39. Bard AJ, Faulkner LR (2001) Electrochemical methods fundamentals and applications, 2nd edn. John Wiley \& Sons Inc., New York

40. Galus Z (1994) Fundamentals of electrochemical analysis, 2nd edn. Ellis Horwood and Polish Scientific Publishers, New York 